\title{
Hello from the Other Side: Can We Perceive Others' Darkness? Observers' Accuracy of the Dark Triad
}

\author{
Lena Lämmle ${ }^{1}$, Fridtjof W. Nussbeck ${ }^{2}$, and Matthias Ziegler ${ }^{3}$ \\ ${ }^{1}$ Faculty of Humanities, Medical School Hamburg, Hamburg, Germany; ${ }^{2}$ Department of Psychology, Universität Konstanz, Konstanz, Germany; \\ ${ }^{3}$ Department of Psychology, Humboldt-Universität zu Berlin, Berlin, Germany
}

\begin{abstract}
The current study builds on research concerning self-other agreement, specifically the TraitReputation-Identity (TRI) Model, and combines this theoretical approach and recent advances from multitrait-multimethod research, specifically the $\mathrm{CTC}(M-1)$ and the latent difference model. This combination was applied to avoid statistical problems associated with latent trait models of the type previously used in TRI Model research. A further aim was to fully exploit the TRI Model's potential by simultaneously modeling more than one trait in one psychometric model. This makes it possible to explore whether raters use similar information to assess different traits or whether this information is observer-specific, as well as whether the traits themselves can shape an individual's Reputation. All analyses were based on a data set capturing the Dark Triad. As the Dark Triad has only rarely been examined from different rater perspectives before, the study also provides new insights into this network of maladaptive traits. A sample of 290 students, their best friends, and one parent were asked to fill out the NPI, Mach IV, and SRP-III. The results suggest that accuracy is high only for narcissism. Moreover, a dark halo could be observed among each of the other-raters for Machiavellianism and psychopathy; target's standings on these two traits were also underestimated by the other-raters. The study's combined use of the TRI Model and modern structural equation methods highlights the usefulness of the TRI Model and adds to the debate about the specific nature of Dark Triad traits.
\end{abstract}

In 2010, Connelly and Ones conducted a meta-analysis on correlations between self- and other-ratings. One of their key findings was that the overlap between different raters and the target (accuracy) is smaller than often assumed. Moreover, the different ratings (i.e. self vs. other) were incrementally valid to each other with regard to selected criteria such as academic success. Based on these findings, Connelly and Ones stated that other-ratings were dramatically underutilized in personality research. Recent years have delivered further evidence that other-ratings add substantial information above and beyond self-ratings for predicting behavior (Poropat, 2014; Ziegler, Danay, Schölmerich, \& Bühner, 2010). This has led to questions concerning the origin of systematic variance in other-ratings. A recently proposed theoretical model offers a straightforward explanation. McAbee and Connelly (2016) proposed the TraitReputation-Identity Model (TRI), which suggests mechanisms explaining the differences as well as congruencies between self- and other-ratings. One key element in this model are the different settings or occasions in which a target him- or herself versus others observe the target's behavior. This is posited to lead to convergence between ratings (Trait) but also to more specific evaluations, sometimes shared by others (Reputation), or unique to the self (Identity). While these developments greatly advance our understanding of why different sources can converge or diverge regarding a target's personality, statistically modeling the TRI Model is not as straightforward. This becomes obvious when looking at recent developments in multitraitmultimethod (MTMM) models (e.g. Eid et al., 2008). These developments not only avoid common problems of MTMM models within a structural equation framework, they also allow for a much more flexible modeling of multi-rater data, thereby opening the door to further insights into the congruence between self- and other-ratings (e.g. Nussbeck, Eid, Geiser, Courvoisier, \& Lischetzke, 2009). Here, we propose to apply these statistical developments to the conceptual framework of the TRI Model. This linking of theory and methods allows us to test some unique assumptions. For example, using such methods allows us to model several traits in the same model, making it possible to test whether the Reputation for one trait shares variance with the Reputation for another trait and thus gauge the information used to rate the traits in question.

Since the seminal work by John and Robins (1993) regarding the role of observability and evaluativeness for

CONTACT Lena Lämmle (ena.laemmle@medicalschool-hamburg.de E Faculty of Humanities, Medical School Hamburg, Am Kaiserkai 1, 20457 Hamburg, Germany. 
self-other agreement, personality research focused on the Big Five. However, in the last decade, personality research has broadened its scope by more strongly considering maladaptive or dark traits (Yalch \& Hopwood, 2016). Among these, the so-called Dark Triad traits are probably the most prominent (Paulhus \& Williams, 2002). Yet, in their metaanalysis, Muris, Merckelbach, Otgaar, and Meijer (2017) pointed out several problems associated with the Dark Triad, such as the strong overlap between scores for the three traits. One of their recommendations was to utilize self- and other-ratings to understand the nomological network of the three traits reflecting the malevolent side of human beings. We answer this call by using data on the Dark Triad and combining the TRI Model with recent developments in MTMM models. The application of the proposed models to the Dark Triad will showcase the advantages of our modeling strategy. In this way, the current research might not only serve as a role model for researchers on how to operationalize the TRI Model but also provides new insights into the Dark Triad itself.

\section{Historical development of research on self-other agreement}

From the 1960s until the 1980s, personality research based on ratings was confronted with several challenges in which other-ratings of personality traits played an important role (Kenrick \& Funder, 1988): The identification of perceptual errors (e.g. the fundamental attribution error or the false consensus effect; McAbee \& Connelly, 2016) and the person-situation debate (Lucas \& Donnellan, 2009) may serve as examples of two prominent challenges. The core of the debate concerned observers' accuracy in making trait judgments. Most commonly, accuracy refers to the convergence between other-ratings and a target's self-ratings (Funder, 1995). The most pertinent questions addressed what attributes the most accurate judges have, how accuracy is affected by situational variations in the target's behavior, and whether accurate judgments are even possible at all (Connelly \& Ones, 2010). An entire body of research sought to provide answers to these questions.

\section{Accuracy}

Connelly and Ones (2010) showed in their meta-analysis that accuracy was lower than expected and varied across traits and different kinds of other-raters. One explanation for this refers to the degree of familiarity between the target and the other-rater; in essence, the higher the familiarity, the higher the accuracy (Connelly \& Ones, 2010; Vazire, 2006; Vazire \& Mehl, 2008). Although there even is some accuracy in cases where the targets and other-raters do not know each other (no familiarity; see Hirschmüller, Egloff, Schmukle, Nestler, \& Back, 2015) as well as between targets and ex situ raters who are neither part of nor affected by the target's circumstances (Rauthmann \& Sherman, 2018), these accuracies are considerably smaller. Hence, there is empirical evidence supporting the claim that accuracy increases with familiarity. This implies that observing the target across time and across situations is one driver of accuracy.

In accordance with the assumptions of Funder's Realistic Accuracy Model (RAM; Funder, 1995) Rogers and Biesanz (2018) recently showed that the target is also a relevant driver of accuracy. This finding supports the notion that even potentially good judges can only be accurate if the target actually displays relevant behavior. Additionally, Hirschmüller and colleagues (2015) showed that observations also need to take place in trait-relevant situations. Thus, there is evidence that accuracy varies according to the observability of the trait being measured, the other-rater's familiarity with the target, and the target's behavior in trait-relevant settings.

\section{Selected models explaining accuracy}

These ideas and results have inspired theory building for quite a long time (e.g. see Cronbach, 1955, for a very early attempt). One of the most prominent models explaining rater accuracy is the previously mentioned RAM (Funder, 1995). According to this model, accuracy depends on the target's opportunity to express the trait in a relevant environment and on the other-rater's ability to perceive the behavior and correctly identify it as trait-relevant. Funder also introduced the idea of the visibility of a trait as the extent to which behavioral cues are available at all. Since then, there have been a variety of suggestions for further specifying the terminology used in the RAM or proposing alternative definitions. Vazire (2010) proposed the self-other knowledge asymmetry (SOKA) model, which proceeds from the assumption that targets and other-raters generally have different knowledge regarding a target's traits. This knowledge asymmetry is seen as influencing accuracy. The knowledge asymmetry itself is posited to depend on observability (also influenced by familiarity) and evaluativeness. Based on her findings Vazire proposed the idea that other-raters might have a biased view of certain targets, especially familiar ones. This idea was further elaborated by Leising, Scherbaum, Locke, and Zimmermann (2015), who suggested an algebraic model for differentiating between the substance of a rating and its variance due to evaluation. Importantly, evaluation is conceptualized here as an interaction between the evaluativeness of the item (behavior) and the evaluative bias of the rater. Thus, the model integrates the evaluativeness ideas of the RAM and the SOKA model.

The aforementioned models and theories imply that the accuracy of ratings (self-other agreement) depends on certain conditions. For self-raters, two conditions have to be met: (i) self-raters must be able to correctly rate their own behavior and/or feelings in principle, and (ii) evaluativeness (self-rater bias) should be low. For other-raters, accuracy depends on four conditions: (i) other-raters must in principle have access to relevant behavior by the target, (ii) targets must exhibit the relevant behavior, (iii) other-raters must be able to correctly identify the relevance of the behavior as a behavioral expression of the trait, and (iv) other-ratings should be unbiased (with respect to sources of 
bias stemming from the rater as well as the items, which reflect more or less desirable behaviors).

Presuming that the fulfillment of these conditions will differ across items, traits, and raters, they should also have an influence on observed ratings. In order to identify the impact of the different conditions, it would be worthwhile to disentangle the variance in observed ratings into its constituent elements, which should reflect (components of) these conditions. This has been achieved in the TRI Model.

\section{Components of self- and other-ratings: The TRI model}

In their TRI Model, McAbee and Connelly (2016) postulate three different systematic components of self- and otherratings: Trait, Reputation, and Identity (see Figure A6, Supplementary material). The Trait should naturally have an impact on all ratings. Identity is conceived as the unique view of self-raters, and Reputation as the shared view of other-raters. Hence, in a factor analytic model, the impact of these different elements can be identified using variance components. The impact of the Trait is reflected in the shared variance across ratings; the impact of Identity is reflected in the systematic residual variance of self-reports (having controlled for the trait); and the impact of Reputation is reflected in the shared variance of other-ratings' systematic residual variance (having controlled for the Trait). Notably, each type of other-rater has its own systematic residual variance, which may overlap more or less strongly across types of raters.

Importantly, the authors suggest that observability drives the associations between indicators and the trait (e.g. the latent variable in a factor analysis). Indicators capturing more observable traits should be highly associated with these traits (show high standardized loading parameters). Indicators capturing less observable traits should depend more strongly on the influences of Identity and Reputation (i.e. show lower standardized loadings on the trait variable). Thus, the model parameters make it possible to gauge the observability of the trait in focus.

Examining the Big Five, McAbee and Connelly (2016) found the highest trait component and thus accuracy in the sense of self-other agreement for extraversion ( $69 \%$ shared variance), followed by conscientiousness (52\%), neuroticism (49\%), agreeableness (44\%), and openness (34\%); the explained variance due to Identity was highest for openness $(34 \%)$, broadly similar for neuroticism, conscientiousness, and agreeableness (ranging from $16 \%$ to $11 \%$ ), and lowest for extraversion (8\%). For Reputation, the lowest explained variance was again observed for extraversion $(8 \%)$, while the other dimensions were quite close to each other (ranging from $14 \%$ to $19 \%)$.

McAbee and Connelly (2016) focused on self-raters as targets and a set of three interchangeable friends resulting in a nested data structure. In their discussion, they pointed to alternative models with differing sampling processes. The current contribution expands on the proposed models by presenting how to analyze non-interchangeable (structurally different) other-raters and by simultaneously modeling multiple traits (i.e. the block design in McAbee and Connelly, 2016 , p. 582). This strategy allows for inspecting the influence of familiarity on the ratings as well as the investigation of rater-specific biases (e.g. halo effect, positivity bias) across traits.

Another important aspect mentioned above was whether or not trait manifestations are visible. High standardized loading patterns reflect high observability. However, at least in the case of the Dark Triad, it might also be relevant to investigate to what extent the trait actually drives the discrepancy between self- and other-ratings. We will show how recent statistical models reflect to which extent a trait actually drives deviations between ratings by others and the self.

\section{The TRI model and the dark triad}

Meta-analyses on the Dark Triad highlight their importance in the personality space (Vize et al., 2018; Muris et al., 2017). They have been shown to have predictive power for various psychosocial phenomena, including aggression, sexrelated issues, interpersonal difficulties, and antisocial tactics (Muris et al., 2017). Recently, Moshagen, Hilbig, and Zettler (2018) suggested a common dark core. Moreover, different saturations for different dark traits were shown, for example narcissism revealed the highest proportion of unique variance whereas Machiavellianism and psychopathy showed higher overlap with this common core. These findings seem to be in line with the most prominent criticism of the Dark Triad, namely the strong overlap between psychopathy and Machiavellianism. Following Muris et al.'s recommendation, in this study, we will use self- and other-ratings to further explore this issue.

Observability and evaluation are seen as important drivers of accuracy. There is evidence that self-other agreement (accuracy) for the Dark Triad traits is comparable to that of Big Five traits (Jones \& Paulhus, 2014). Moreover, Küfner, Dufner, and Back (2015) showed that the dark traits manifest themselves in observable behavior and that others can perceive these manifestations. However, both of these studies used relatively small samples of other-raters as well as relatively short measures. Thus, we utilized longer measures in a considerably larger sample.

As for the Big Five, familiarity should be generally influential with regard to the observability of the Dark Triad traits. However, it is also reasonable to assume that observability differs across other-raters, even when all allegedly know a target well (e.g. parents vs. friends). Prior research on narcissism shows that narcissistic behavior seems unaffected by situational changes (Maaß \& Ziegler, 2017). Moreover, Maaß, Lämmle, Bensch, and Ziegler (2016) reported that similarity in narcissism explains personality similarity between friends. This implies that narcissism might not only be more observable in general compared to the other Dark Traits, but also that it might be especially observable by close friends. For psychopathy, it can be assumed that psychopathy-related behavior might be easier to observe for friends than for parents. Psychopathy is 
related to reckless, antisocial behavior with a temporal focus (Jones \& Paulhus, 2014), which is assumed to occur with greater likelihood among friends (Moffitt, 1993). In line with this, accuracy in psychopathy ratings has been reported to be higher for ratings by friends than parents (Yalch \& Hopwood, 2016). Finally, Machiavellianism is especially interesting as an able Machiavellian should be able to influence others without them realizing that they are being played (Wilson, Near, \& Miller, 1996). Thus, observability might potentially be lowest for Machiavellianism which would result in lower accuracy. We will present models that allow for an integrative examination of these assumptions.

To sum up, the TRI Model proposes that observability differs according to how well a target is known by others, or more specifically, from which situations the target is known. Specific hypotheses regarding the observability of manifestations of Dark Triad traits for friends and parents can be made on the basis of these assumptions. We assume that psychopathy is more observable for friends. Machiavellianism should be least observable for both parents and friends. Finally, narcissism should be the most observable trait. In turn, these assumed differences in observability should be reflected in differing accuracies and specificity in ratings (i.e. influence of Identity and Reputation).

\section{Modeling the TRI model}

McAbee and Connelly's (2016) modeling approach can be categorized as a variant of a correlated-trait-correlatedmethod (CTCM) model. In both the CTCM and the TRI Model, the trait is considered to influence all observations. The systematic residuals of the TRI Model (Identity and rater-specific systematic residuals) correspond to method effects in the CTCM model. The only difference between TRI Model and CTCM models is that the Identity factor (method factor for self-reports) must not correlate with the Reputation factor and that the associations between the other-raters' method factors are presumed to follow a unidimensional structure (Reputation). This modeling approach with adequate parameter restrictions as reported by McAbee and Connelly fits the study design of targets and a set of interchangeable raters.

Yet, this approach is problematic for several reasons (Eid, 2000) when raters are not considered interchangeable. First, it has been shown that CTCM models often yield improper solutions or are subject to identification problems. Second, interpretations of the trait and method factors are problematic. In the CTCM model, all indicators of a trait are related to all other indicators of that trait via their loading parameters on the latent trait variable, yet they are also related to all other indicators of that trait via their loadings on their respective method factor and the correlation between the method factors. Hence, whether a given trait variable reflects all trait influences and whether method effects also encompass trait influences remains unclear. The situation in the TRI Model with interchangeable raters is quite similar: The Trait is associated with all trait-specific indicators via their trait-loading parameters. Yet, Reputation is also related to almost all indicators except for the self-report. If there are strong associations between the other-reports and only weak associations with the self-report, both the Trait and the Reputation factor will most strongly depend on the otherreports, leading to exactly the same problem as that concerning the meaning of the latent variables in a CTCM model. Additionally, scores for Trait, Identity, and Reputation as well as their interpretations can change within the same data set when different methods are used (see Geiser, Eid, West, Lischetzke, \& Nussbeck, 2012). That is, the very same individual will have different trait scores if the TRI Model is estimated with self-report, a parent report, or with a friend report as well.

In the remainder of this contribution, we will adapt two specific multitrait-multimethod (MTMM) models proposed by Eid and colleagues (2008) and illustrate their interpretation with respect to the conceptual TRI Model for structurally different raters (i.e. self-raters, parents, and friends). The correlated trait-correlated method (minus 1) model [CTC(M-1)] and the Latent Difference MTMM model (LD model) provide remedies for the problems encountered with the CTCM model. Moreover, modeling multiple traits simultaneously will also allow for within-other-rater residual correlations between different traits, across-rater residual correlations within and across traits, and, in the LD model, the influence of the trait itself on other-raters' deviations from the self-rating, which may deepen our understanding of interrater congruence.

We will conceptually introduce the $\operatorname{CTC}(M-1)$ and the LD models (formal model definitions can be found in Table A3, Supplementary material) using the example of the Dark Triad (narcissism, psychopathy, and Machiavellianism assessed by self-raters, one parent, and one friend). We define the self-reports as the outstanding method. We presume that targets are best informed about their behaviors in all kinds of situations, know when they try to deceive people in their environment, and when they openly display their motives, including in communication. Targets may try to hide their Dark Triad traits from their social surroundings, making it difficult for other-raters to make valid judgments. For example, targets with high scores on Machiavellianism should rarely be seen as such, as they succeed in inconspicuously manipulating others.

\section{CTC $(M-1)$ model}

The CTC $(M-1)$ model (Figure 1) should be applied in research focusing on convergence (agreement or accuracy). In this model, the trait is modeled as a latent factor underlying the response variables in the reference method (selfreports); hence, the trait variable (e.g. NAR) in the upper

\footnotetext{
'Raters who are structurally different differ with respect to their perspective on and available information about the trait. Psychometrically, different raters (here targets, friends, and parents) do not belong to the same population of raters. In this case, it is most meaningful to contrast methods with one another, choose one method as the reference method (Eid et. al, 2008), and model either a correlated trait-correlated method minus 1 (CTC(M-1)) model (Eid et al., 2003) or a Latent Difference (LD) model (Pohl, Steyer, \& Kraus, 2008)
} 


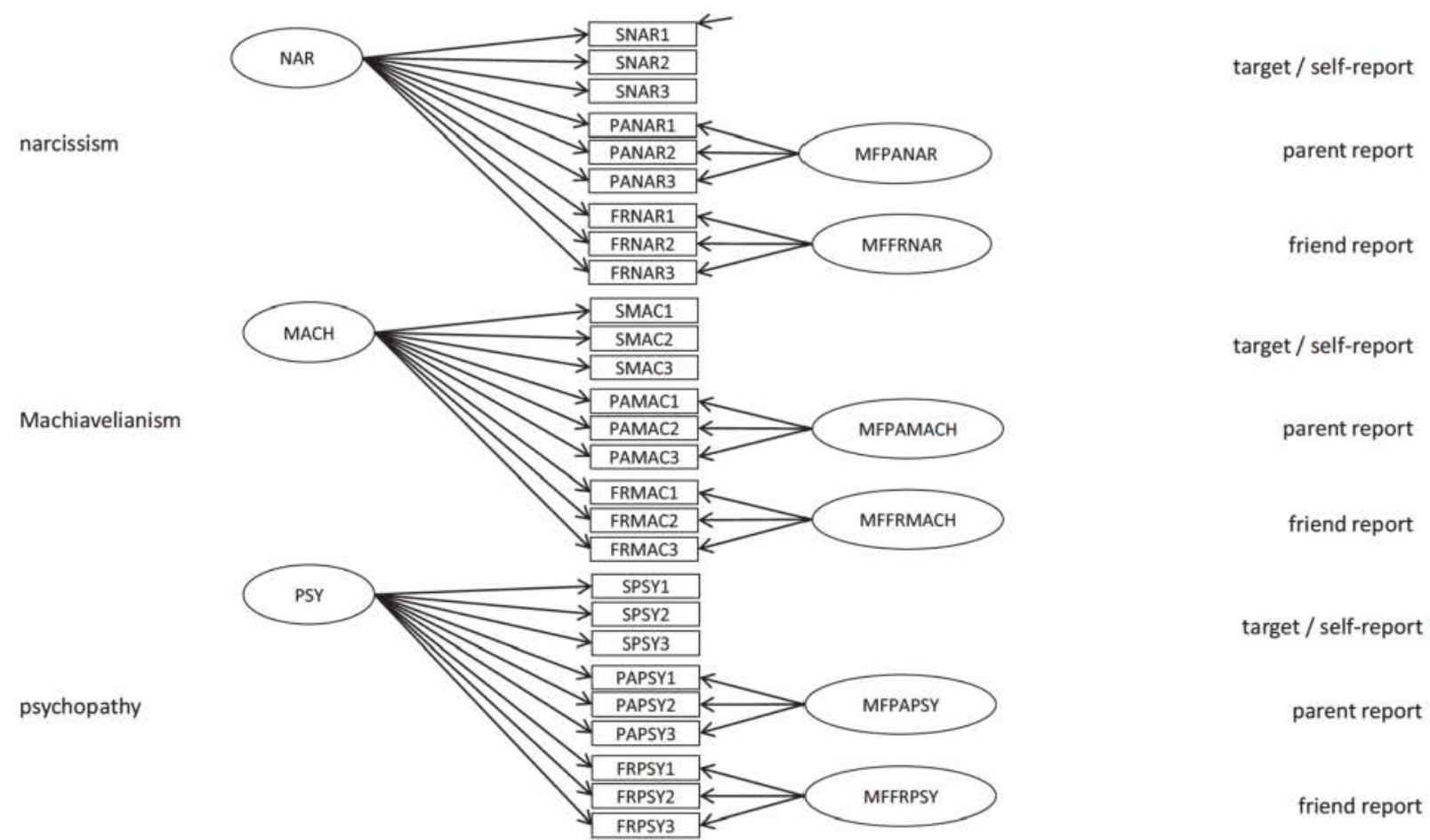

Figure 1. CTC(M-1) model. NAR: narcissism, MACH: Machiavellianism; PSY: psychopathy; MFPANAR, MFPAMACH, MFPAPSY: method factors for parent reports; MFFRNAR, MFFRMACH, MFFRPSY: method factors for friend reports; SNAR1 to FRPSY3: indicators (3 per trait and method combination); For the sake of accessibility, the measurement residual is only depicted for SNAR1 but estimated for all indicators; trait variables may correlate (not depicted), all method factors may correlate (not depicted), method factors may correlate with traits not belonging to the same trait-method-unit.

part of Figure 1 reflects narcissism measured by self-report. There is no method factor for the targets' self-reports. Consequently, the latent factor does not change in meaning if other-raters are added to or removed from the model.

Response variables from friends' (and parents') reports load on this trait factor; in other words, friend reports are "predicted" using the self-report trait factor. The systematic deviation of the friend report indicators from this prediction (the association of the residuals) is captured in the method factor (MF) for the friend report (FR) for narcissism (NAR). As a residual, the method factor for the friend report (MFFRNAR) must not correlate with the trait factor (NAR). Hence, friend report scores are additively decomposed in a predicted part using the self-report trait score as predictor, the systematic deviation from this prediction (method factor), and measurement error.

Choosing the self-report as reference method implies that Trait and Identity cannot be disentangled. Hence, the trait in the CTC $(M-1)$ model is a combination of Trait and Identity in the TRI. The method factors are residual factors and can be understood as source-specific Reputation factors (friend-specific or parent-specific Reputation).

Due to the additive decomposition of other-report scores, the CTC $(M-1)$ model allows for the estimation of variance components (see Eid et al., 2003; see also Table 1). The consistency coefficient (Con) indicates the amount of explained error-free variance in other-reports. It reflects the agreement between other-raters and the target (accuracy). The methodspecificity coefficient $(M S)$ reflects the amount of error-free variance that is due to source-specific variance. The two coefficients sum up to 1 and can be directly compared to illuminate the degree to which other-ratings reflect either the (self-reported) trait or unique views.

\section{Latent difference model}

While the focus of the CTC $(M-1)$ model is on analyzing convergence between self- and other-ratings, the LD model focuses on analyzing differences between self- and otherratings, again with the self-report chosen as reference method. The latent trait factor in the LD model (e.g. NAR) is the trait measured by self-report (see Table A3, Supplementary material), just as in the CTC $(M-1)$ model. The friend report indicators are regressed on this trait factor with restricted loading parameters. That is, the first indicator of the friend report must exhibit the same loading parameter and the same intercept as the first indicator of the self-report (indicated by "a" in Figure A7, Supplementary material). This restriction implies that the expected score of a friend report indicator is the same as the expected score of the corresponding self-report indicator. The systematic deviation of the friend report indicators from the self-report indicators is captured in the latent difference variable (LDFRNAR). The LD variable's mean value indicates whether friends generally over- or underestimate the trait score provided by the targets. Interindividual differences between friends in their over- or underestimation are represented by the variance in the LD factor. 
Table 1. Standardized loading parameters, reliability, consistency, and method-specificity coefficients for indicators of the CTC(M-1) model for the dark triad.

\begin{tabular}{|c|c|c|c|c|c|c|c|c|c|}
\hline & \multicolumn{3}{|c|}{ Trait loading parameters } & \multicolumn{3}{|c|}{ Method factor loading parameters } & \multirow[b]{2}{*}{ Reliability } & \multirow[b]{2}{*}{ Consistency } & \multirow[b]{2}{*}{ Method-Specificity } \\
\hline & $i$ & SE & Two-Tailed $p$-value & $i$ & SE & Two-Tailed $p$-value & & & \\
\hline \multicolumn{10}{|l|}{ Narcissism } \\
\hline SNAR1 & .88 & .02 & $<.01$ & & & & .77 & 1 & \\
\hline SNAR2 & .92 & .02 & $<.01$ & & & & .85 & 1 & \\
\hline SNAR3 & .89 & .04 & $<.01$ & & & & .79 & 1 & \\
\hline PANAR1 & .75 & .03 & $<.01$ & .50 & .04 & $<.01$ & .81 & .69 & .31 \\
\hline PANAR2 & .73 & .03 & $<.01$ & .53 & .04 & $<.01$ & .81 & .65 & .35 \\
\hline PANAR3 & .71 & .05 & $<.01$ & .49 & .04 & $<.01$ & .75 & .68 & .32 \\
\hline FRNAR1 & .81 & .03 & $<.01$ & .43 & .04 & $<.01$ & .84 & .78 & .22 \\
\hline FRNAR2 & .77 & .03 & $<.01$ & .52 & .04 & $<.01$ & .86 & .69 & .31 \\
\hline FRNAR3 & .82 & .04 & $<.01$ & .41 & .04 & $<.01$ & .84 & .80 & .20 \\
\hline \multicolumn{10}{|c|}{ Machiavellianism } \\
\hline SMAC1 & .70 & .04 & $<.01$ & & & & .48 & 1 & \\
\hline SMAC2 & .66 & .05 & $<.01$ & & & & .43 & 1 & \\
\hline SMAC3 & .74 & .04 & $<.01$ & & & & .55 & 1 & \\
\hline PAMAC1 & .30 & .06 & $<.01$ & .68 & .04 & $<.01$ & .56 & .16 & .84 \\
\hline PAMAC2 & .34 & .06 & $<.01$ & .57 & .05 & $<.01$ & .44 & .26 & .74 \\
\hline PAMAC3 & .32 & .06 & $<.01$ & .77 & .04 & $<.01$ & .69 & .15 & .85 \\
\hline FRMAC1 & .23 & .07 & $<.01$ & .63 & .05 & $<.01$ & .45 & .12 & .88 \\
\hline FRMAC2 & .33 & .06 & $<.01$ & .62 & .05 & $<.01$ & .49 & .22 & .78 \\
\hline FRMAC3 & .40 & .06 & $<.01$ & .69 & .04 & $<.01$ & .63 & .25 & .75 \\
\hline \multicolumn{10}{|c|}{ psychopathy } \\
\hline SPSY 1 & .85 & .02 & $<.01$ & & & & .73 & 1 & \\
\hline SPSY2 & .87 & .02 & $<.01$ & & & & .75 & 1 & \\
\hline SPSY3 & .88 & .02 & $<.01$ & & & & .77 & 1 & \\
\hline PAPSY1 & .47 & .05 & $<.01$ & .74 & .03 & $<.01$ & .76 & .29 & .71 \\
\hline PAPSY 2 & .46 & .05 & $<.01$ & .76 & .03 & $<.01$ & .76 & .27 & .73 \\
\hline PAPSY 3 & .51 & .05 & $<.01$ & .76 & .03 & $<.01$ & .79 & .31 & .69 \\
\hline FRPSY1 & .61 & .04 & $<.01$ & .67 & .04 & $<.01$ & .84 & .45 & .55 \\
\hline FRPSY2 & .58 & .04 & $<.01$ & .68 & .04 & $<.01$ & .80 & .42 & .58 \\
\hline FRPSY 3 & .55 & .04 & $<.01$ & .71 & .04 & $<.01$ & .80 & .38 & .62 \\
\hline
\end{tabular}

Note. SNAR1 to SNAR3, SMACH1 to SMACH3 and SPP1 to SPP3 represent self-report indicators for narcissism, Machiavellianism and psychopathy; PA represents parent report indicators and FR represents friend report indicators.

\section{Correlations in the MTMM models}

The CTC(M-1) and LD models differ with respect to their allowed correlations (see Table A4, Supplementary material; see Eid et al., 2008, Pohl et al., 2008 for a deeper discussion):

i. In the CTC $(M-1)$ model, all trait variables may correlate, reflecting the association between self-reported Dark Triad traits. Moreover, all rater-specific method factors may correlate across traits and across raters. Correlations of method factors across traits within a single type of rater indicate the generalizability (e.g. halo effect) of over- or underestimation for a type of rater (e.g. parents may tend to judge their offspring consistently lower or higher on the Dark Triad traits). Correlations of method effects across raters (e.g. MFPANAR with MFFRNAR) indicate the degree to which over- and underestimation generalizes across raters for a given trait (indicating that similar information is used/Reputation). Method factors may also correlate across traits and raters. The correlation between MFFRMACH and MFPAPSY indicates whether targets rated higher than predicted by their friends on Machiavellianism are rated higher (lower) than predicted by their parents on psychopathy. Finally, traits may correlate with method factors belonging to other traits. That is, MACH may correlate with MFFRPSY, for example, indicating that targets scoring high on
Machiavellianism are rated higher than expected on psychopathy by friends.

ii. In the LD model, all trait variables may correlate, reflecting the association between self-reported Dark Triad traits. Moreover, all rater-specific method factors may correlate across traits and across raters. Correlations across traits within a single type of rater indicate whether raters consistently show smaller or larger deviations across traits, that is, a positive correlation between LDFRMACH and LDFRPSY, for example, indicates that friends who produce larger deviations than the average on Machiavellianism also do so for psychopathy. Correlations across types of raters within traits (e.g. LDFRMACH and LDPAMACH) indicate whether targets who are judged higher on Machiavellianism by their friends than in the self-report are also judged higher on Machiavellianism by their parents. Correlations between traits and LD factors belonging to other traits indicate whether targets scoring high on Machiavellianism are rated higher on psychopathy by their friends than by themselves, for example (correlation between MACH and LDFRPSY). Most interestingly, the correlation between a trait variable and the LD variable for the same trait provides specific insight into rater agreement. Assume a negative correlation between MACH and LDFRMACH. This correlation would reflect that targets who give themselves high scores on Machiavellianism are rated lower 
by their friends on Machiavellianism. In other words, such a correlation would reveal that "good manipulators" are able to successfully hide their Machiavellian manipulating behavior from others. In summary, these correlations shed light on the information used, potential halo or social desirability (Ziegler \& Bühner, 2009) effects and the ways in which the traits themselves are related to these.

\section{Method}

\section{Sample and procedure}

Two hundred and ninety participants were recruited as targets $(56 \%$ female). This sample size was chosen due to the fact that the main focus of TRI Model analyses is on correlations, which have been shown to stabilize at a sample size of 250 (Schönbrodt \& Perugini, 2013), and that the CTC $(M-1)$ model converges at sample sizes of 250 (Nussbeck, Eid, \& Lischetzke, 2006), even with categorical indicators. The mean age was $21.44(S D=1.81)$. The mean age of the best friend sample was $22.85(S D=4.54 ; 49 \%$ female) and the mean age of the parent sample was 51.22 $(S D=6.74 ; 72 \%$ mothers). Before beginning the study, informed consent was obtained from the students, one of their parents, and their best friend in order to avoid the complete loss of self- and other-ratings. Consequently, none of the participants needed to be excluded during data collection. The questionnaires for all students were administered during a university course. At the end of the course, students were given the questionnaires for their best friend and a parent to fill in. Friends and parents could either send the questionnaires to a postal address or hand the tests to the target participants in a sealed envelope. Thus, we could not control for careless responding among the other-raters (possible consequences are discussed in the limitations section). However, we used the Mahalanobis distance using scale scores to identify multivariate outliers (see A0, Supplementary material). There were no strong deviations. The total sample size amounted to 870 . After all participants had filled in the measures, they were debriefed and thanked for their participation.

\section{Test materials}

Narcissism was assessed with the German version (Schütz, Marcus, \& Sellin, 2004) of the Narcissistic Personality Inventory (NPI; Raskin \& Terry, 1988), a 40-item forcedchoice measure of subclinical narcissism (Paulhus \& Williams, 2002) with comparable psychometric properties in both languages (Jakobwitz \& Egan, 2006; Schütz et al., 2004). In each item, one of the two statements reflects a narcissistic attitude ("I can read people like a book"), while the other does not ("People are sometimes hard to understand"). Machiavellianism was assessed with the German version (Rauthmann, 2013) of the Machiavellianism questionnaire Mach IV, consisting of 20 items with a 5-point rating scale ranging from $1=$ "strongly disagree" to
$5=$ "strongly agree" (Christie \& Geis, 1970). Participants rated statements such as "There is no excuse for lying to someone else" or "It is wise to flatter important people". Previous studies revealed good psychometric properties (e.g. Hansen \& Hansen, 1991), but also some limitations with regard to the factor structure in both languages (Rauthmann, 2013) which we tried to address by a parceling procedure (see below). Psychopathy was assessed with the Self-Report Psychopathy Scale-III (SRP-III), consisting of 64 items with a 5 -point rating scale ranging from $1=$ "strongly disagree" to $5=$ "strongly agree" (Paulhus, Hemphill, \& Hare, 2009). Example items are "Most people are wimps" or "I'm not tricky or sly". The psychometric quality of this German version has been shown in other studies as well (e.g. Rauthmann, 2012, Rauthmann, 2013 ).

In the other-ratings of the Dark Triad measures, the standardized stimulus material was changed slightly. Instead of describing oneself, participants were asked to describe their daughter, son or friend, and the item pronouns were changed from "I" to "he/she". Overall, we encountered very little missing data, with a total of six missing entries for narcissism, seven missing entries for Machiavellianism, and eight missing entries for psychopathy (all in parent reports). We decided to run all statistical models using only complete cases $(N=279)$ and compare the model results to the results applying the robust full-information maximum likelihood (FIMLR) estimator to the full sample $(N=279)$. There turned out to be only very slight differences in model parameters $(<0.01$ for unstandardized parameters); hence, we report the FIMLR results.

\section{Statistical analyses}

All statistical models were estimated with Mplus 7.4 (Muthén \& Muthén, 1998-2017) using a robust maximum likelihood estimator (MLR/FIMLR). We provide annotated input files in the Supplementary material (A1). Model fit is assessed using standard criteria: a non-significant chi-square value, $\mathrm{CFI} \geq .97$, RMSEA $\leq .06$; SRMR $<.05$ (SchermellehEngel, Moosbrugger, \& Müller, 2003). In order to locally identify the MTMM models, we decided to create three homogeneous test parcels per trait for every method (Cole, Perkins, \& Zelkowitz, 2016); see Supplementary Material A2 for more details on the parceling procedure). We followed a stepwise modeling procedure in which we first estimated trait-specific models for narcissism, Machiavellianism, and psychopathy separately. This strategy allows for easily identifying and fixing local model misspecifications. We provide the correlation matrix as well as means and standard deviations for all parcels in the Supplementary material (A5).

\section{Results}

Following the stepwise modeling procedure, it turned out that the three indicators of narcissism did not load onto a single latent variable. The model for narcissism did not converge, indicating problems with the method factor loadings. Restricting the method factor loadings did not remedy the 
Table 2. Correlations for the CTC(M-1) (upper part) and the LD MTMM Model (lower part).

\begin{tabular}{|c|c|c|c|c|c|c|c|c|c|c|}
\hline & NAR1 & NAR2 & NAR3 & MACH & PSY & MFPA NAR & MFFRNAR & MFPA MACH & MFFR MACH & MFPA PSY \\
\hline \multicolumn{11}{|c|}{ CTC(M-1) Model } \\
\hline NAR2 & .77 & & & & & & & & & \\
\hline NAR3 & .71 & .74 & & & & & & & & \\
\hline $\mathrm{MACH}$ & .22 & .22 & .30 & & & & & & & \\
\hline PSY & .42 & .42 & .40 & .68 & & & & & & \\
\hline MFPANAR & $x$ & $x$ & $x$ & .08 & .06 & & & & & \\
\hline MFFRNAR & $x$ & $x$ & $x$ & .08 & .08 & .55 & & & & \\
\hline MFPA MACH & .22 & .18 & .12 & $x$ & .09 & .10 & -.11 & & & \\
\hline MFFR MACH & .23 & .16 & .08 & $x$ & .14 & .03 & -.01 & .32 & & \\
\hline MFPAPSY & .08 & .14 & .08 & -.05 & $x$ & .10 & -.07 & .74 & .27 & \\
\hline MFFRPSY & .04 & .05 & .04 & -.02 & $\mathrm{x}$ & .00 & .03 & .26 & .78 & .34 \\
\hline \multicolumn{11}{|c|}{ LD MTMM Model } \\
\hline & NAR & MACH & PSY & LDPA NAR & LDFR NAR & LDPA MACH & LDFR MACH & LDPA PSY & M & $\sigma^{2}$ \\
\hline NAR & & & & & & & & & 0.36 & .02 \\
\hline MACH & .28 & & & & & & & & 0.06 & .01 \\
\hline PSY & .48 & .69 & & & & & & & 0.04 & .01 \\
\hline LDPANAR & -.21 & .02 & -.04 & & & & & & 3.41 & .12 \\
\hline LDFRNAR & -.06 & .08 & .05 & .54 & & & & & -0.40 & .19 \\
\hline LDPAMACH & .05 & -.39 & -.19 & .05 & -.15 & & & & -0.17 & .17 \\
\hline LDFRMACH & .04 & -.47 & -.20 & -.03 & -.06 & .44 & & & 2.30 & .10 \\
\hline LDPAPSY & -.08 & -.31 & -.39 & .09 & -.08 & .72 & .31 & & -0.26 & .10 \\
\hline LDFRPSY & -.08 & -.20 & -.27 & .00 & .02 & .29 & .73 & .41 & -0.07 & .08 \\
\hline
\end{tabular}

Note. SNAR1 to SNAR3, SMAC1 to SMAC3 and SPSY1 to SPSY3 represent self-report indicators for narcissism, Machiavellianism and psychopathy; PA represents parent report indicators and FR represents friend report indicators. LDPANAR, latent difference-parents for narcissism; LDFRNAR, latent difference-friends for narcissism; LDPAMACH, latent difference-parents for Machiavellianism; LDFRMACH, latent difference-friends for Machiavellianism; LDPAPSY, latent differenceparents for psychopathy; LDFRPSY, latent difference-friends for psychopathy.

problem; hence, we decided to model three latent (parcelspecific) factors for narcissism. This model exhibited good fit $\quad\left(\chi^{2}=31.00 ; \quad d f=23 ; \quad p=.12 ; \quad\right.$ RMSEA $=.04 ; \quad 90 \%$ C.I. $=[.00 ; .06] ;$ CFI $>.99 ;$ SRMR $=.06)$. The model with one trait variable for Machiavellianism $\left(\chi^{2}=37.08\right.$; $d f=20$; $p=.01 ; \quad$ RMSEA $=.06 ; \quad 90 \%$ C.I. $=[.03 ; .08] ; \quad$ CFI $=.98 ;$ $\mathrm{SRMR}=.03$ ) fit acceptably well, and the model with only one trait variable for psychopathy $\left(\chi^{2}=89.14 ; d f=20\right.$; $p<.01 ; \quad$ RMSEA $=.11 ; \quad 90 \% \quad$ C.I. $=[.09 ; \quad .14] ; \quad$ CFI $=.97 ;$ SRMR $=.02$ ) had mixed fit statistics. We hence decided to model three latent factors for narcissism and one latent factor each for Machiavellianism and psychopathy.

\section{CTC(M-1) model: Convergence of self- and other-ratings}

Figure A8 (see Supplementary material) depicts the CTC $(M-1)$ model which fit the data acceptably well $\left(\chi^{2}=500.87\right.$; $d f=261 ; \quad p<.01 ; \quad$ RMSEA $=.06 ; \quad 90 \% \quad$ C.I. $=[.05 ; \quad .06] ;$ $\mathrm{CFI}=.96 ;$ SRMR $=.04$ ). Table 1 presents the standardized loading parameters for the trait and method factors. All loading parameters were statistically significant $(p<.05)$. For narcissism and psychopathy, we found high standardized loading parameters, indicating that the traits accounted for a large portion of the observed variance in the self-report indicators (also reflected in the reliability estimates in Table 1). For Machiavellianism, the standardized loading parameters for the self-ratings were less pronounced and the indicators had lower reliability estimates.

For other-reports, we found two different patterns: For narcissism, there were high standardized loading parameters $\left(1^{\text {st }}\right.$ part of Table 1$)$, indicating strong associations between the latent trait and its friend and parent report indicators. For Machiavellianism and psychopathy $\left(2^{\text {nd }}\right.$ and 3 rd parts of Table 1), the standardized loading parameters were much smaller. The standardized loading parameters can be interpreted as correlations between the latent factor and the indicator (Eid, Lischetzke, Nussbeck, \& Trierweiler, 2003) representing the overlap between error free self-ratings and other ratings with measurement error (comparable to trait influences in the TRI Model). An error free estimation of this correlation is provided by the square root of the Consistency Coefficient (see Table 1).

Our second important finding with respect to standardized loading parameters is that the method-factor loadings exhibited an opposite pattern. We found strong loadings for Machiavellianism and psychopathy (2nd and 3rd part of Table 1), but low loadings for narcissism indicators (1st part of Table 1) on their respective method factors. Hence, shared observer-specific effects and thus observer-specific Reputation were smaller for narcissism than for Machiavellianism and psychopathy.

Table 1 also presents the reliability estimates for the indicators, consistency, and method-specificity coefficients. Consistency coefficients for self-report indicators are 1 by definition. The complete amount of error-free variance is due to the trait factor. For the other-report indicators, we found the same pattern described above: Relatively high consistencies (65-80\%) and low method specificities (sourcespecific Reputation; 20-35\%) for narcissism and lower consistencies (ranging from $12 \%$ to $45 \%$ ) and larger method specificities (ranging from $55 \%$ to $88 \%$ ) for Machiavellianism and psychopathy.

Table 2 (upper part) shows the correlation coefficients of the latent variables in the $\operatorname{CTC}(M-1)$ model. In the upper left (in light gray), correlations between the three indicatorspecific narcissism traits show that these variables were strongly related $(.71 \leq r \leq .77)$. Nevertheless, the three indicators were too heterogeneous to form a single trait variable, 
as indicated by the model fit indices. Machiavellianism and psychopathy were moderately related to narcissism $(.22 \leq r$ $\leq .40)$; Machiavellianism and psychopathy were strongly related to one another $(r=.68)$.

The correlations of the method factors (in gray, lower right triangle) include a moderate to strong correlation between the method factors for narcissism for parents and friends $(r=.55)$. This can be seen as reflecting the general Reputation for this trait as defined in the TRI Model. The correlation between the method factors for parents and friends was $r=.32$ for Machiavellianism and $r=.34$ for psychopathy. Thus, the shared variance of the two otherreports was smaller for Machiavellianism and psychopathy than for narcissism. This indicates that, as expected, the specific views of parents and friends differ more strongly for these traits than for narcissism. However, parents and friends do agree to a greater extent than they agree with the targets for all three traits, as reflected by the correlations between the method factors. Thus, there is Reputation in the sense of the TRI Model for all three dark traits.

The correlations of the method factors for Machiavellianism and psychopathy within parents $(r=.74)$ and within friends $(r=.78)$ were large. This implies that each observer relies on similar information when judging psychopathy and Machiavellianism. Thus, there is a sourcespecific Reputation across Machiavellianism and psychopathy within parents and within friends, representing a halo effect or bias. However, this halo effect is rater specific and does not generalize across other-raters, as correlations between friends' method factors for Machiavellianism and parents' method factors for psychopathy and vice versa were not significant. Interestingly, this generalized Reputation across Machiavellianism and psychopathy is not related to the specific Reputation for narcissism, as shown by the very low correlation between the narcissism method effects and the corresponding Machiavellianism and psychopathy method effects for parents and friends $(-.07<r<.10$, n.s. $)$.

\section{LD model: Differences between self- and other-ratings}

The LD model with 2nd order factor for narcissism (see Figure A9, Supplementary material) also fit the data acceptably well $\quad\left(\chi^{2}=556.33 ; \quad d f=297 ; \quad p<.01 ; \quad \chi^{2} / d f<2\right.$; RMSEA $=.06 ; 90 \%$ C.I. $=[.05 ; .06]$; CFI $=.95$; SRMR $=.04$ ). The standardized loading parameters of the LD MTMM model exhibited a comparable pattern as in the $\operatorname{CTC}(M-1)$ model (not depicted). In order to provide a meaningful interpretation of the latent difference variables, we additionally introduced a 2 nd order latent trait for narcissism. The last but one column of the lower part of Table 2 depicts the mean values of the latent variables with similar findings compared to previous research (e.g. Maaß et al., 2016). The mean score for narcissism was low, with the targets endorsing around 14 narcissistic statements on average $(0.36 \times 40=14.4)$; average Machiavellianism scores (3.41) were close to the theoretical scale mean (mean of a 1 to 5 rating scale $=3$ ), and psychopathy average scores (2.30) fell below the theoretical scale midpoint (3). Interestingly, parents and friends exhibited only a small degree of bias with respect to narcissism in terms of the endorsed items: Parents tended to rate their child's narcissism 0.06 units higher $(0.06 \times 40=2.4$ statements $)$, and friends tended to rate the targets' narcissism 0.04 units higher $(0.04 \times 40=1.6$ statements). However, these differences correspond to 0.29 and 0.43 standard deviation units of the 2 nd order trait and may hence be considered small to moderate effects. That is, both parents and friends rated the targets as slightly more narcissistic on average than the targets rated themselves. The opposite was true for Machiavellianism and psychopathy. Both parents and friends underestimated Machiavellianism and psychopathy on average. Most strikingly, parents underestimated their child's Machiavellianism by almost half a point on the scale (LDPAMACH $=-0.41$ ). They also underestimated their child's psychopathy (LDPAPSY $=-0.27)$ - descriptively, they showed a larger tendency to underestimate their child's Machiavellianism and psychopathy than friends did (both LD mean scores for parents are more extreme than for friends; LDFRMACH $=-0.17$ and LDFRPSY $=-0.07$ ). This slightly stronger congruence between friends and targets supports the idea that negative behavior is more likely to be exhibited with friends around than with parents. This notion is also supported by the larger variances in parents' LD factors (last column of Table 2), representing larger differences in the parent ratings. In fact, as expected, there was only a small difference between targets and friends for psychopathy.

Table 2 (lower part) shows the correlations of the latent variables in the $\mathrm{LD}$ model. Overall, the results are very similar to the correlations for trait and method factors found in the CTC $(M-1)$ model (light gray and gray parts). However, and most importantly, difference variables and trait variables may correlate in the LD model. These correlations revealed that highly narcissistic individuals (high self-report scores) were rated less narcissistic by their parents which is indicated by the negative correlation between self-reported narcissism and the difference variable for parents $(r=-.21)$. However, this was not true for friends $(r=-.06$, n.s.). Highly Machiavellian individuals were rated less Machiavellian by their parents $(r=-39)$ and friends $(r=-.47)$ than they rated themselves. The same was found for psychopathy (target-parent correlation: $r=-.31$ and target-friend correlation: $r=-.20$ ). Psychopathic individuals were judged less psychopathic by their parents $(r=-.39)$ and friends $(r=-.27)$ as well as less Machiavellian by their parents and friends $(r=-.19$ and $r=-.20)$. Hence, the LD model reveals that targets scoring higher on Machiavellianism may be better able to hide the undesirable parts of their dark side (Machiavellianism itself but also psychopathy) from their parents and friends.

\section{Discussion}

The aim of the present study was to extend the statistical modeling strategies for the TRI Model (McAbee \& Connelly, 2016) by applying CTC $(M-1)$ and LD models to multiple(non-interchangeable) rater data. More specifically, we 
aimed to test whether familiarity drives accuracy and to what extent the kind of situations shared with the target influence accuracy. Moreover, we focused on the Dark Triad traits, which have not been the subject of much research on self-other agreement. The results reveal a high agreement between other- (parent and friend) and self-ratings for narcissism, but low agreement for Machiavellianism and psychopathy. The modeling approach allowed us to uncover specific halo effects affecting Machiavellianism and psychopathy other-ratings. The findings also suggest that friends and targets might base their judgments on different kinds of observations or on different situations encountered with the target. Finally, the notion of a "good" Machiavellian (i.e. one who is not easily detected) was supported.

\section{Implications for the TRI model}

The present methodological approach makes it possible to model source-specific Reputations and test the influence of the traits themselves on these Reputations. Moreover, estimations of self-other agreement (convergence) as well as the degree to which others share specific views can be explored. Convergence between other-raters is indicative of good observability. Discrepancies between other-raters highlight the importance of being part of a target's life in specific circumstances (i.e. moments being relevant for trait manifestations). Thus, accuracy might be driven by observability, which comes with familiarity. However, the observability of specific trait manifestations seems to be driven by the kind of relationship the other-rater has with the target and thereby the kinds of situations encountered together. Thus, what is important is not familiarity per se but rather the kinds of situations familiarity allows others to share with the target.

\section{Self-other agreement for the dark triad}

As predicted, narcissism seems to be the most observable trait of the Dark Triad. The relatively high consistency coefficient indicates strong self-other agreement and, hence, also a strong similarity in other-ratings. Together with prior findings on the situation-independent manifestation of narcissism (Maaß \& Ziegler, 2017) the notion of observability as a driver of accuracy is supported. Moreover, the method factors for friends and parents in the $\mathrm{CTC}(M-1)$ model for narcissism correlated more strongly than the method factors for Machiavellianism and psychopathy. This correlation represents the degree to which parents and friends share a specific view of the target's narcissism after controlling for the (self-reported) trait and, therefore, parallel the Reputation aspect of the TRI Model. Following the logic of the TRI Model, friends' and parents' specific views regarding narcissism might be due to the same behaviors exhibited by the respective targets. Here, from the perspective of narcissism manifestation, familiarity seems to lead others into similar situations with the targets; in other words, narcissism seems to manifest itself in the same way with different kinds of others (Maaß \& Ziegler, 2017).
For the other two dark traits, the method factor correlations across sources and thus the Reputations were considerably smaller. This indicates that parents and friends use less similar information when rating those traits or that they tend to interpret these pieces of information differently. However, the correlations between the method factors for psychopathy and Machiavellianism within friends and parents were relatively strong. Thus, there is a dark halo effect within each kind of other-rater for Machiavellianism and psychopathy (low discriminant validity in MTMM logic). Importantly, as pointed out above, this dark halo is not caused by the same kind of information used to rate narcissism. Thus, in the terms of the TRI Model, narcissism Reputation seems to be more rater-unspecific than Machiavellian and psychopathy Reputation. These findings are in line with findings by Moshagen and colleagues (2018) who postulated a dark core generally underlying dark traits and found that narcissism was less influenced by this core than other dark traits.

The LD models revealed that while narcissism is relatively accurately assessed by both parents and friends, parents tend to underestimate both other Dark Triad traits whereas friends only underestimate Machiavellianism. More importantly, the LD model showed that higher Machiavellianism and higher psychopathy trait scores lead to an underestimation of these traits by both parents and friends. We interpret this finding as support for the notion of a "good" Machiavellian, which means a person who is able to deceive and manipulate others without them knowing it. An alternative explanation could be that we are simply observing an effect of liking. Parents and friends like the target and therefore rate these negative traits in a more positive light (see Leising, Erbs, \& Fritz, 2010; Leising, Ostrovski, \& Zimmermann, 2013; Vazire, 2006; Vazire \& Mehl, 2008). Interestingly, though, the relations between traits and deviations were rater-specific and did not occur for narcissism. This speaks against a mere positivity bias, which is likely to be rater-unspecific and would therefore increase the correlation between the other-rater specific variances (Ziegler \& Bühner, 2009). Future research should include other-raters who know but dislike the target or otherraters who do not really know the target well to further explore the idea of a "good" Machiavellian.

These findings are also interesting in light of the widespread assumption that high familiarity with the target, which both friends and family members experience to a similar extent, should lead to similar accuracies (Connelly \& Ones, 2010). Despite the large overlap with the self-reports, the current results highlight that Reputation and thus the use of similar information by others can be low. Hence, there are differences between parent and friend ratings. This emphasizes the need to use different kinds of other-raters to capture potential other-rater differences as well as the need to carefully plan multi-rater studies if aggregating across different kinds of other-raters is planned.

Thus, the current results show that the Dark Triad traits do not yield comparable self-other agreement estimates. As assessed in the CTC $(M-1)$ model and in line with our theoretical assumptions, self-other agreement was strong for narcissism and rather low for Machiavellianism and 
psychopathy, which points to higher observability for narcissism (Connelly \& Ones, 2010; Yalch \& Hopwood, 2016) compared to psychopathy and Machiavellianism. Our findings further support this hypothesis and, on a conceptual basis, are informative with regard to the nomological network of the Dark Triad traits themselves.

\section{Insights into the dark triad from modeling trait, reputation, and identity using the $\mathrm{CTC}(M-1)$ and LD models}

This study's findings show that there is a stronger Reputation amongst friends and parents for narcissism than for psychopathy and Machiavellianism. At the same time, however, the correlations between the Machiavellianism and psychopathy method factors within each source were strong. This means that while parents and friends might use different information when rating the target, each source seems to use similar information when rating the two different traits. Thus, the very source-specific Reputations for these dark traits cast a dark halo onto each other. Meta-analytic findings on the nomological network of the Dark Triad indicate a strong overlap between psychopathy and Machiavellianism in self-reports (Vize, Lynam, Collison, \& Miller, 2016). Our multi-rater findings provide further support for this overlap, with a strong correlation of $r=.68$ between the two traits in the CTC(M-1) model. Importantly, though, the LD model showed that both other-raters tend to underestimate the target's trait level for psychopathy and especially for Machiavellianism. Thus, the dark halo does not necessarily imply lower ratings, it simply shows that the information used to rate these two traits is similar within each source.

As just pointed out, the LD model provided unique insights. Leising, Gallrein, and Dufner (2014) suggested that those who know a person better have a more positive view than the person him- or herself. The current research shows that, for the Dark Triad, this seems to apply more to parents than to friends. Moreover, it could also be shown that this underestimation varies depending on the dark trait in focus: The strongest underestimation occurred for Machiavellianism, specifically among parents, followed by psychopathy. Whether this means that parents have distorted views of their offspring or whether they just lack opportunities to observe corresponding behaviors remains an open question. The current findings do show, however, that friends, on average, do not tend to strongly underestimate their friends' own views of their dark sides.

Additionally, the LD approach made it possible to estimate the influence of the traits themselves on over- and underestimations. Again, the different Dark Triad traits exhibited differing effects. Stronger narcissism had only a small negative correlation with the LD score for parents, showing that higher narcissism might lead to an underestimation of the same trait by parents. This could mean that the parent's positive view of their child (Leising et al., 2014) increases the more the trait manifests. Another explanation might be the behavioral-genetic profiles of the Dark Triad traits. Even though all three have a moderate to large heritable component, Machiavellianism also has environmental roots (Vernon, Villani, Vickers, \& Harris, 2008). Thus, the clear genetic component of narcissism along with its high visibility for the self and others might positively affect parents' judgments of their narcissistic child. This might also relate to the ego-reinforcement motive (Jones \& Paulhus, 2014) of the narcissistic parent him- or herself. All other correlations between LD scores and narcissism were either not significant or rather small (not exceeding $r=.05$ ).

For Machiavellianism, the results of the LD model show that higher trait scores led parents and friends to underestimate Machiavellianism and psychopathy. Thus, targets high on Machiavellianism might be more able to hide this trait from their friends and family, which is in line with the clear strategic orientation of this trait (Jones \& Paulhus, 2014) Similar, yet less pronounced, results occurred for psychopathy. Considering the dark halo described above, this raises the question of what kind of information, meaning what kind of trait manifestations, drive this underestimation.

Another explanation for the trait influence on the latent differences might be that children, in their adult lives, hide their Machiavellian and psychopathic tendencies from their parents for reasons such as respect. Or it might be that people's tendency to hide our dark side is lower in contacts with peers. This interpretation is in line with Yalch and Hopwood's (2016) assumption that aggressive behavior mostly occurs among friends.

All in all, the current findings support our assumption that familiarity is an important factor driving differences in rater accuracy. Moreover, our findings also imply that mere familiarity does not necessarily lead to better observability of traits, as previously assumed. It can safely be assumed that both parents and friends have a close relationship with the target. Yet this only led to accuracy and a shared Reputation for narcissism. For Machiavellianism and psychopathy, the Reputation was lower, indicating differences in the kind of behavior observed between other-raters despite their familiarity. At the same time, the findings also show that parents and friends do not utilize the same information when rating the targets' Machiavellianism and psychopathy. Thus, which source is tapped as the other-rater matters.

Finally, this is the first study to show that the trait itself can influence Reputation. The findings show that higher Machiavellianism and psychopathy scores lead to an underestimation of these same traits by other-raters. Whether this is related to observability or is caused by a more specific mechanism cannot be answered with the current data. It would be insightful for future studies to collect experience sampling data from self- and other-ratings in the same situation along with situation descriptions. However, the fact that there is a source-specific halo makes it likely that reduced observability is not the only reason for lower agreement between parents and friends.

\section{Recommendations for statistical modeling of multiple rater dato}

Perceiving different types of raters as a special case of assessment methods enables researchers to make use of the 
findings from state-of-the-art research on MTMM models (see Eid et al., 2008, for a thorough discussion) as already discussed above. The most pertinent question concerns the structure of the methods in the analysis:

1. If raters differ from each other with respect to their access to motives, feelings, and thoughts, the CTC $(M-1)$ or LD models are appropriate choices. The choice of a reference method is not trivial and has an impact on the interpretation of the model parameters. The operationalization we chose here results in a Trait definition by the self-rating. As discussed above, this also means that Trait and Identity cannot be separated. If, for example, the parent ratings were chosen as reference method, the interpretation of the latent variables in the model would change. The Trait would now be defined by the parent-ratings which in turn would not have a method factor. The residuals for self- and friend-ratings would reflect differences from the scores predicted by the parent-ratings and thus how much the target's own view and the friend's view differ from the parents view. The correlation between those two would not reflect a Reputation but rather how congruent targets and friends are in their deviation from the parents' view. Thus, McAbee and Connelly's use of the Johari window would no longer be applicable. In other words, when combining the TRI and CTC(M-1) models it is necessary to use self-ratings as reference method in order to maintain the interpretations for the constituent parts of the TRI.

2. Researchers can choose between the CTC $(M-1)$ or LD model in accordance with their research question. If the focus is on variance components such as trait, Identity, and Reputation, the CTC $(M-1)$ model should be estimated, as consistency and method-specificity coefficients can be estimated. If the focus is on differences between scores and hence on absolute agreement, the LD model is the best choice.

\section{Limitations}

One limitation of the current study is that the analyses are based on a specific sample of target participants recruited from psychology courses; whether our results generalize to the general population remains unclear. In addition, we could not control ratings for careless responding, meaning that the variance might be restricted and thus the effects potentially underestimated. Although the sample size was sufficiently large to soundly estimate the MTMM models (see Nussbeck et al., 2006, for comparable models using ordinal indicators), we lack power to test mean differences between the LD factors in the LD models. Furthermore, we did not differentiate between mother and father, which might have increased the variance in parent-ratings. Besides, we used a very specific set of measures. Our exclusive reliance on the NPI for narcissism might have led to a strong emphasis on the grandiose side of narcissism, leaving the vulnerable side unexplored (see Crowe et al., 2019).
Whether the findings can be generalized to this functionally more different side of narcissism remains an open research question. Another limitation is that even though the German version of the SRP-III used here has been used in several other studies (e.g. Rauthmann, 2013), no psychometric evaluation for th German translation has been published yet. Finally, we used item parcels as indicators in the MTMM models to reduce model complexity. This allowed us to focus on the specificities of the MTMM modeling approach. Future studies with a more substantive focus on the dark triad (and larger samples) should rather use items as indicators in order to gain a deeper understanding of the pieces of information (i.e. the item wording) raters use to judge a target's trait and the extent to which items overlap.

\section{Conclusions}

The combination of the logic of McAbee and Connelly's (2016) TRI framework with the CTC $(M-1)$ and LD models enables the consideration of several traits simultaneously (here narcissism, Machiavellianism, psychopathy), with the results revealing that narcissism Reputation seems to be more source-unspecific than Machiavellian and psychopathy Reputation. Moreover, utilizing the suggested methodological approaches makes it possible to gauge the nature of the information used by other-raters. Accordingly, we found that parents and friends each use similar information when rating Machiavellianism and psychopathy, but different incidents and behaviors when rating narcissism. However, the results also imply that familiarity per se does not lead to the use of identical information by other-raters. In other words, familiarity is very likely source-specific and a generalization of results from one type of other-rater to another seems not feasible. Furthermore, the traits themselves were shown to drive a person's Reputation: Parents of children with a stronger dark side underestimated all three traits; for best friends, this was only true for Machiavellianism and psychopathy. Finally, the findings provide further insights into the criticized overlap between Machiavellianism and psychopathy.

\section{References}

Christie, R. C., \& Geis, F. L. (1970). Studies in Machiavellianism. New York: Academic Press.

Cole, D. A., Perkins, C. E., \& Zelkowitz, R. L. (2016). Impact of homogeneous and heterogeneous parceling strategies when latent variables represent multidimensional constructs. Psychological Methods, 21(2), 164-174. doi: $10.1037 /$ met0000047

Connelly, B. S., \& Ones, D. S. (2010). Another perspective on personality: Meta-analytic integration of observers' accuracy and predictive validity. Psychological Bulletin, 136(6), 1092-1122. doi:10.1037/ a0021212

Cronbach, L. J. (1955). Processes affecting scores on "understanding of others" and "assumed similarity. Psychological Bulletin, 52(3), 177-193, doi: 10.1037/h0044919

Crowe, M. L., Lynam, D. R, Campbell, W, K., \& Miller, J. D. (2019). Exploring the structure of narcissism: Towards an integrated solution. Journal of Personality. doi:10.31219/osf.io/wpcjq

Eid, M. (2000). A multitrait-multimethod model with minimal assumptions. Psychometrika, 65(2), 241-261. doi:10.1007/BF02294377 
Eid, M., Lischetzke, T., Nussbeck, F. W., \& Trierweiler, L. I. (2003) Separating trait effects from trait-specific method effects in multitrait-multimethod models: A multiple-indicator CT-C(M-1) model. Psychological Methods, 8(1), 38-60. doi:10.1037/1082-989X.8.1.38

Eid, M., Nussbeck, F. W., Geiser, C., Cole, D. A., Gollwitzer, M., \& Lischetzke, T. (2008). Structural equation modeling of multitrait multimethod data: Different models for different types of methods. Psychological Methods, 13(3), 230-253. doi:10.1037/13219

Funder, D. C. (1995). On the accuracy of personality judgment: A realistic approach. Psychological Review, 102(4), 652-670.

Geiser, C., Eid, M., West, S. G., Lischetzke, T., \& Nussbeck, F. W. (2012). A comparison of method effects in two confirmatory factor models for structurally different methods. Structural Equation Modeling, 19(3), 409-436.

Hansen, C. H., \& Hansen, R. D. (1991). Constructing personality and social reality through music: Individual differences among fans of punk and heavy metal music. Journal of Broadcasting \& Electronic Media, 35(3), 335-350. doi:10.1080/08838159109364129

Hirschmüller, S., Egloff, B., Schmukle, S. C., Nestler, S., \& Back, M. D. (2015). Accurate judgments of neuroticism at zero acquaintance: A question of relevance. Journal of Personality, 83(2), 221-228. doi:10. 1111 /jopy.12097

Jakobwitz, S., \& Egan, V. (2006). The dark triad and normal personality. Personality and Individual Differences, 40(2), 331-339. doi:10. 1016/j.paid.2005.07.006

John, O. P., \& Robins, R. W. (1993). Determinants of interjudge agreement on personality traits: The Big Five domains, observability, evaluativeness, and the unique perspective of the self. Journal of Personality, 61(4), 521-551. doi:10.1111/j.1467-6494.1993.tb00781.x

Jones, D. N., \& Paulhus, D. L. (2014). Introducing the short Dark Triad (SD3): A brief measure of dark personality traits. Assessment, 21(1), 28-41. doi:10.1177/1073191113514105

Kenrick, D. T., \& Funder, D. C. (1988). Profiting from controversy: Lessons from the person-situation debate. American Psychologist 43(1), 23, doi:10.1037/0003-066X.43.1.23

Küfner, A. C. P., Dufner, M., \& Back, M. D. (2015). Das Dreckige Dutzend und die Niederträchtigen Neun. Diagnostica, 61(2), 76-91. doi:10.1026/0012-1924/a000124

Leising, D., Erbs, J, \& Fritz, U. (2010). The letter of recommendation effect in informant ratings of personality. Journal of Personality and Social Psychology, 98(4), 668-682.

Leising, D., Gallrein, A. M. B., \& Dufner, M. (2014). Judging the behavior of people we know: Objective assessment, confirmation of preexisting views, or both? Personality \& Social Psychology Bulletin, 40(2), 153-163. doi:10.1177/0146167213507287

Leising, D., Ostrovski, O., \& Zimmermann, J. (2013). “Are We Talking About the Same Person Here?": Interrater Agreement in Judgments of Personality Varies Dramatically With How Much the Perceivers Like the Targets. Social Psychological and Personality Science, 4(4), 468-474.

Leising, D., Scherbaum, S., Locke, K. D., \& Zimmermann, J. (2015). A model of "substance" and "evaluation" in person judgments. Journal of Research in Personality, 57, 61-71. doi:10.1016/j.jrp.2015.04.002

Lucas, R. E., \& Donnellan, M. B. (2009). Editorial: If the person-situation debate is really over, why does it still generate so much negative affect? Journal of Research in Personality, 43(2), 146-149. doi:10.1016/ j.jrp.2009.02.009

Maaß, U., Lämmle, L., Bensch, D., \& Ziegler, M. (2016). Narcissists of a feather flock together: Narcissism and the similarity of friends. Personality and Social Psychology Bulletin, 42(3), 366-384. doi:10. $1177 / 0146167216629114$

Maaß, U., \& Ziegler, M. (2017). Narcissistic self-promotion is not moderated by the strength of situational cues. Personality and Individual Differences, 104, 482-488. doi:10.1016/j.paid.2016.09.008

McAbee, S. T., \& Connelly, B. S. (2016). A multi-rater framework for studying personality: The Trait-Reputation-Identity model. Psychological Review, 123(5), 569-591. doi:10.1037/rev0000035

Moffitt, T. E. (1993). Adolescence-limited and life-course-persistent antisocial behavior: A developmental taxonomy. Psychological Review, 100(4), 674-701. doi:10.1037/0033-295X.100.4.674
Moshagen, M., Hilbig, B. E., \& Zettler, I. (2018). The dark core of personality. Psychological Review, 125(5), 656-688. doi:10.1037/rev0000111

Muris, P., Merckelbach, H., Otgaar, H., \& Meijer, E. (2017). The malevolent side of human nature: A meta-analysis and critical review of the literature on the dark triad (narcissism, Machiavellianism, and psychopathy). Perspectives on Psychological Science, 12(2), 183-204. doi: $10.1177 / 1745691616666070$

Muthén, L. K., \& Muthén, B. O. (1998-2017). Mplus user's guide (8th ed.). Los Angeles, CA: Muthén \& Muthén.

Nussbeck, F. W., Eid, M., Geiser, C., Courvoisier, D. S., \& Lischetzke, T. (2009). A CTC $(M-1)$ model for different types of raters. Methodology, 5(3), 88-98. doi:10.1027/1614-2241.5.3.88

Nussbeck, F. W., Eid, M., \& Lischetzke, T. (2006). Analysing multitrait-multimethod data with structural equation models for ordinal variables applying the WLSMV estimator: What sample size is needed for valid results? The British Journal of Mathematical and Statistical Psychology, 59(1), 195-213. doi:10.1348/000711005X67490

Paulhus, D. L., Hemphill, J., \& Hare, R. (2009). Manual for the selfreport psychopathy scale (SRP-III). Toronto: Multi-Health Systems.

Paulhus, D. L., \& Williams, K. M. (2002). The Dark Triad of personality: Narcissism, Machiavellianism, and psychopathy. Journal of Research in Personality, 36(6), 556-563. doi:10.1016/S0092-6566(02)00505-6

Pohl, S., Steyer, R., \& Kraus, K. (2008). Modelling method effects as individual causal effects. Journal of the Royal Statistical Society: Series A, 171, 1-23.

Poropat, A. E. (2014). Other-rated personality and academic performance: Evidence and implications. Learning and Individual Differences, 34, 24-32. doi:10.1016/j.lindif.2014.05.013

Raskin, R., \& Terry, H. (1988). A principal-components analysis of the Narcissistic Personality Inventory and further evidence of its con struct validity. Journal of Personality and Social Psychology, 54(5), 890. doi:10.1037//0022-3514.54.5.890

Rauthmann, J. F. (2012). Towards multifaceted Machiavelliansim: Content, factorial, and validity of a German Machiavellianism Scale. Personality and Individual Differences, 52(3), 345-351. doi:10.1016/j. paid.2011.10.038

Rauthmann, J. F. (2013). Investigating the MACH-IV with items response theory and proposing the timed $\mathrm{MACH}^{*}$. Journal of Personality Assessment, 95(4), 388. doi:10.1080/00223891.2012.742905

Rauthmann, J. F., \& Sherman, R. A. (2018). The description of situations: Towards replicable domains of psychological situation characteristics. Journal of Personality and Social Psychology, 114(3), 482-488. doi:10.1037/pspp0000162

Rogers, K. H., \& Biesanz, J. C. (2018). Reassessing the good judge of personality. Journal of Personality and Social Psychology, 117(1), 186-200. Advance online publication. doi:10.1037/ pspp0000197

Schermelleh-Engel, K., Moosbrugger, H., \& Müller, H. (2003). Evaluating the Fit of Structural Equation Models: Tests of Significance and Descriptive Goodness-of-Fit Measures. Methods of Psychological Research, 8(2), 23-74.

Schönbrodt, F. D., \& Perugini, M. (2013). At what sample size do correlations stabilize? Journal of Research in Personality, 47(5), 609-612. doi:10.1016/j.jrp.2013.05.009

Schütz, A., Marcus, B., \& Sellin, I. (2004). Die Messung von Narzissmus als Persönlichkeitskonstrukt. Diagnostica, 50(4) 202-218. doi:10.1026/0012-1924.50.4.202

Vazire, S. (2006). Informant reports: A cheap, fast, and easy method for personality assessment. Journal of Research in Personality, 40(5), 472-481. doi:10.1016/j.jrp.2005.03.003

Vazire, S. (2010). Who knows what about a person? The self-other knowledge asymmetry (SOKA) model. Journal of Personality and Social Psychology, 98(2), 281. doi:10.1037/a0017908

Vazire, S., \& Mehl, M. R. (2008). Knowing me, knowing you: The accuracy and unique predictive validity of self-ratings and otherratings of daily behavior. Journal of Personality and Social Psychology, 95(5), 1202. doi:10.1037/a0013314

Vernon, P. A., Villani, V. C., Vickers, L. C., \& Harris, J. A. (2008). A behavioral genetic investigation of the Dark Triad and the Big Five. 
Personality and Individual Differences, 44(2), 445-452. doi:10.1016/j. paid.2007.09.007

Vize, C. E., Collison, K. L., Miller, J. D., \& Lynam, D. R. (2018). Examining the effects of controlling for shared variance among the Dark Triad using meta-analytic structural equitation modeling. European Journal of Personality, 32(1), 46-61.

Vize, C. E., Lynam, D. R., Collison, K. L., \& Miller, J. D. (2016). Differences among Dark Triad components: A meta-analytic investigation. Personality Disorders: Theory, Research, and Treatment, 9(2), 101-111. doi: $10.1037 /$ per0000222
Yalch, M. M., \& Hopwood, C. J. (2016). Target-, informant-, and meta-perceptual ratings of maladaptive traits. Psychological Assessment, 29(9), 1142-1156. doi:10.1037/pas0000417

Ziegler, M., \& Bühner, M. (2009). Modeling socially desirable responding and its effects. Educational and Psychological Measurement, 69(4), 548-565. doi:10.1177/0013164408324469

Ziegler, M., Danay, E., Schölmerich, F., \& Bühner, M. (2010). Predicting academic success with the Big Five rated from different points of view: Self-rated, other rated and faked. European Journal of Personality, 24, 341-355. doi:10.1002/per.753 